\title{
ATRIBUTOS FÍSICO-HÍDRICOS DE UM LATOSSOLO DE CERRADO EM DIFERENTES POSIÇÕES DE AMOSTRAGEM NA LAVOURA CAFEEIRA ${ }^{(1)}$
}

\author{
Ivoney Gontijo ${ }^{(2)}$, Moacir de Souza Dias Junior ${ }^{(3)}$, Paulo Tácito Gontijo \\ Guimarães $^{(4)}$ \& Cezar Francisco Araujo-Junior ${ }^{(5)}$
}

\begin{abstract}
RESUMO
A compactação do solo, ocasionada por diferentes intensidades de tráfego, pode provocar danos à estrutura do solo, limitando a produção em lavouras cafeeiras. O objetivo deste trabalho foi desenvolver modelos de capacidade de suporte de carga para um Latossolo sob Cerrado, cultivado com cafeeiros, bem como investigar os efeitos do manejo da lavoura cafeeira na distribuição de poros e na retenção de água, em diferentes posições na lavoura cafeeira. $O$ estudo foi realizado em um Latossolo Vermelho distrófico típico localizado nas coordenadas de $18^{\circ} 59,15$ " $\mathrm{S}$ e $46^{\circ} 56^{\prime} 47$ " W, cultivado com cafeeiros (Coffea arabica L.) em espaçamento de 4 x $1 \mathrm{~m}$, implantado no ano de 1995 no município de Patrocínio, MG. As diferentes posições de amostragem na lavoura cafeeira foram: projeção da saia (PS), linha de tráfego (LT) e centro da entrelinha (EL). Além disso, amostras indeformadas também foram coletadas na mata nativa (MN) para obter os modelos de capacidade de suporte de carga. Em cada posição de amostragem foram coletadas amostras deformadas e indeformadas na profundidade de $10-13 \mathrm{~cm}$, para avaliação dos seguintes atributos físico-hídricos: pressão de preconsolidação $\left(\sigma_{\mathrm{p}}\right)$, umidade gravimétrica (U), densidade do solo (Ds), porosidade total (PT), macroporosidade (Ma), microporosidade (Mi), curva de retenção de água no solo, teor de matéria orgânica (MO), textura, densidade de partículas e limites de liquidez, plasticidade e contração. Os resultados obtidos sugerem que a capacidade de suporte de carga do Latossolo decresceu na seguinte ordem: $\mathrm{LT}>\mathrm{MN}=\mathrm{PS}>\mathrm{EL}$. O solo sob a posição EL foi mais suscetível à compactação em virtude da menor capacidade de suporte de carga, devido a uma subsolagem realizada meses antes da amostragem, reduzindo a sua resistência mecânica. O solo sob MN e a PS apresentaram mesma capacidade de suporte de carga, indicando a manutenção de uma estrutura
\end{abstract}

\footnotetext{
(1) Parte da Tese de Doutorado do primeiro autor apresentada ao Departamento de Ciência do Solo da Universidade Federal de Lavras - UFLA. Recebido para publicação em janeiro de 2008 e aprovado em outubro 2008.

(2) Doutor em Solos e Nutrição de Plantas. Centro de Ciências Agrárias da Universidade Federal do Espírito Santo - UFES. Caixa Postal 16, CEP 29500-000 Alegre (ES). Bolsista PNPD/CAPES. E-mail: ivoneygontijo@yahoo.com.br

(3) Professor Associado do Departamento de Ciência do Solo da Universidade Federal de Lavras - UFLA. Caixa Postal 3037, CEP 37200-000 Lavras (MG). Bolsista CNPq. E-mail: msouzadj@ufla.br

(4) Pesquisador da Empresa de Pesquisa Agropecuária de Minas Gerais - EPAMIG/CTSM. Caixa Postal 176, CEP 37200-000 Lavras (MG). Bolsista FAPEMIG. E-mail: paulotgg@ufla.br

(5) Doutorando em Solos e Nutrição de Plantas do Departamento de Ciência do Solo, UFLA. Bolsista CNPq. E-mail: cfaj@bol.com.br
} 
favorável para o crescimento do sistema radicular do cafeeiro. A água disponível foi maior para o solo nas posições EL e PS e menor para a LT e MN; o solo sob a LT mantém maior microporosidade em relação à EL desestruturada pela subsolagem.

Termos de indexação: ensaio de compressão uniaxial, pressão de preconsolidação, estrutura do solo, água no solo, Coffea arabica $\mathrm{L}$.

\title{
SUMMARY: PHYSICAL-HYDRIC ATTRIBUTES OF AN OXISOL FROM THE CERRADO REGION UNDER COFFEE PLANTATION AS AFFECTED BY THE SAMPLING POSITION
}

\begin{abstract}
The soil compaction, induced by different traffic intensities, can cause damage in the soil structure, reducing the coffee plantation production. The objectives of this study was to develop a model of load support capacity for a Red Latosol (Oxisol) under Cerrado and under coffee and to investigate the effect of coffee plantation management on soil pore distribution and soil water retention, in different sampling positions in the coffee plantation. The stud site is located at $18^{\circ} 59^{\prime} 15$ " $\mathrm{S}$ and to $46^{\circ} 56^{\prime} 47$ " W Patrocínio county, Minas Gerais State, and has been cultivated with coffee (Coffea arabica L.), at a $4 \times 1 \mathrm{~m}$ spacing, since 1995. The different sampling positions in the coffee plantation were: under canopy (PS), cart track (LT) and in the middle of the interrows (EL). Besides, samples were also collected in a native cerrado vegetation (MN) to obtain the soil load capacity models. Disturbed and undisturbed soil samples were collected at each sampling point at a depth of 10-13 cm to assess the following physical-hydric attributes: preconsolidation pressure $\left(\sigma_{p}\right)$, gravimetric moisture content $(U)$, bulk density $(D s)$, total porosity $(P T)$, macropores (Ma), micropores (Mi), soil water retention curve, organic matter (MO), texture, particle density and liquid, plastic, and contraction limits. The results obtained suggest that the load support capacity of the soil decreases in the following order: $L T>M N=P S>E L$. The EL position was most susceptible to soil compaction due to lower load support capacity associated with subsoiling just before sampling, decreasing the soil mechanical resistance. The soil load support capacity under MN and PS was homogeneous, indicating a soil structure favorable to the development of the coffee root system. The available water in the soil was higher in EL and PS and lower in LT and MN positions. The micropores of soil under $L T$ were larger than in the EL, which was disturbed by the subsoiling.
\end{abstract}

Index terms: uniaxial compression test, preconsolidation pressure, soil structure, soil porosity, soil water, Coffea arabica $L$.

\section{INTRODUÇÃO}

A busca por alternativas que proporcionem a sustentabilidade do ambiente agrícola, principalmente do ponto de vista da manutenção da estrutura do solo, tem sido constante devido à crescente ocorrência do processo de compactação. A maioria das ocorrências do processo de compactação do solo na agricultura moderna deve-se ao tráfego de implementos agrícolas, o qual é parte integrante do sistema de manejo das culturas. O aumento do tamanho dos implementos agrícolas e a alta freqüência de tráfego são causas importantes da indução da compactação e deterioração da estrutura do solo (Lipiec \& Hatano, 2003).

Estima-se que aproximadamente 68 milhões de hectares em todo o mundo estejam em processo de degradação devido à compactação, sendo o tráfego de máquinas agrícolas responsável pela maior parte das causas de compactação nos solos agrícolas (Flowers $\&$ Lal, 1998). Esse problema tende a se intensificar em razão da tendência de aumento do tamanho e da potência dos implementos agrícolas, por permitir economia, trabalhar em larga escala, além de reduzir os custos de mão-de-obra (Yavuzcan et al., 2004).

Em lavouras cafeeiras com a implantação em áreas aptas à mecanização e cultivo em larga escala, a utilização de máquinas agrícolas e a degradação da estrutura do solo têm sido observadas com maior freqüência. Outro aspecto a ser considerado é que a área de trabalho para mecanização em lavouras cafeeiras é restrita a pequenas faixas nas entrelinhas de plantio, o que obriga as máquinas a transitarem sempre no mesmo local (Carvalho Filho et al., 2004) e próximo à saia do cafeeiro, local onde se concentram as suas raízes ativas. 
Estudos recentes indicam que alterações estruturais nas entrelinhas dos cafeeiros podem alterar a condutividade hidráulica do solo e, conseqüentemente, as taxas de infiltração de água durante chuvas ou irrigação (Timm et al., 2005, 2006). Além disso, o manejo de plantas invasoras (Araujo-Junior et al., 2008), o tráfego eventual de máquinas (Silva et al., 2006) e as operações de subsolagem promovem alterações da capacidade de suporte de carga do solo (CSC) e, conseqüentemente, de sua resistência à compactação.

A compactação causada pelo tráfego de máquinas pode alterar a distribuição de poros por tamanho e, por conseguinte, alterar a retenção e a distribuição de água no solo (Dias Junior, 2000). Devido à constante alteração no sistema poroso do solo proporcionada pelo manejo de lavouras cafeeiras, a curva de retenção de água no solo pode ser um indicativo dos efeitos do manejo na distribuição de poros por tamanho, das propriedades hidráulicas, bem como do armazenamento de água no solo, já que essa curva é sensivelmente alterada pela estrutura do solo (Hillel, 1970). Diante do exposto, observa-se que pela forma da curva de retenção de água no solo é possível fazer inferências sobre os efeitos do manejo no solo e dos ciclos de umedecimento e secagem na distribuição de poros por tamanho (Pires et al., 2008), assim como avaliar a qualidade estrutural desse solo (Dexter, 2004). Entretanto, neste estudo, a avaliação da qualidade estrutural foi feita pelos modelos de capacidade de suporte de carga, considerando que a pressão de preconsolidação é considerada como a maior pressão aplicada ao solo no passado, e esta divide a curva de compressão em duas regiões de deformações: elásticas e recuperáveis e plásticas e irrecuperáveis (Dias Junior \& Pierce, 1996; Dias Junior, 2000). Assim, a pressão de preconsolidação é considerada um indicador quantitativo da resistência interna do solo e da sua sustentabilidade estrutural.

O objetivo do presente trabalho foi desenvolver modelos de capacidade de suporte de carga para um Latossolo sob Cerrado e cultivado com cafeeiros em diferentes posições de amostragem na lavoura cafeeira, bem como investigar os efeitos do manejo da lavoura cafeeira sob a distribuição de poros e na retenção de água no solo.

\section{MATERIAL E MÉTODOS}

O estudo foi realizado em uma lavoura cafeeira (Coffea arabica L.) implantada no ano de 1995, localizada no município de Patrocínio, MG. Anteriormente à implantação da lavoura cafeeira a área foi utilizada com pastagem. A área total do experimento é de $40.000 \mathrm{~m}^{2}$, com cafeeiros implantados no espaçamento de $4 \times 1 \mathrm{~m}$. A área encontra-se a $18^{\circ} 59^{\prime} 15$ " de latitude sul e a $46^{\circ} 56^{\prime} 47$ " de longitude, a oeste de Greenwich e a uma altitude de $934 \mathrm{~m}$.
O clima da região, segundo a classificação de Köppen, é do tipo Aw, com duas estações bem definidas, inverno seco e verão chuvoso, e precipitação pluvial média anual de $1.620 \mathrm{~mm}$ (Silva \& Malvino, 2005). A vegetação predominante da região é do tipo Cerrado. O relevo é plano a suave ondulado, com declividade média de $3 \%$, sendo o solo classificado como Latossolo Vermelho distrófico típico LVd (Embrapa, 2006), apresentando $780 \mathrm{~g} \mathrm{~kg}^{-1}$ de argila, $110 \mathrm{~g} \mathrm{~kg}^{-1}$ de silte e $110 \mathrm{~g} \mathrm{~kg}^{-1}$ de areia.

A análise granulométrica foi realizada pelo método da pipeta (Day, 1965), utilizando como dispersante químico $10 \mathrm{~mL}$ de $\mathrm{NaOH} 1 \mathrm{~mol} \mathrm{~L}^{-1}$, em contato com a amostra durante $24 \mathrm{~h}$; a dispersão mecânica foi feita utilizando o processo de ultra-som, devido à forte agregação encontrada no Latossolo estudado. Foi utilizado um aparelho operando a $20 \mathrm{kHz}$, com potência aplicada de 60 Watts, conforme técnicas calorimétricas descritas por Sá et al. (2000).

$\mathrm{O}$ controle de plantas invasoras foi realizado antes das adubações, com a aplicação de herbicida de pósemergência na projeção da copa do cafeeiro e nas entrelinhas, em associação com roçadora. $\mathrm{O}$ controle fitossanitário foi feito uma vez ao mês, e a adubação de cobertura, aplicada de $40 \mathrm{em} 40$ dias no período entre outubro e fevereiro de cada ano.

A amostragem foi realizada em janeiro de 2006, na profundidade de $10-13 \mathrm{~cm}$; coletaram-se 30 amostras indeformadas em anéis metálicos de $2,54 \mathrm{~cm}$ de altura por $6,30 \mathrm{~cm}$ de diâmetro, para as seguintes posições na lavoura cafeeira: centro das entrelinhas (EL), linha de tráfego (LT) e projeção da saia (PS), bem como na condição de mata nativa (MN), utilizada como referência dos atributos físicos. Essas amostras foram usadas para construção do modelo de capacidade de suporte de carga do solo. Foram coletadas, ainda, seis amostras de cada local para obtenção das curvas de retenção de água, de acordo com o método da Embrapa (1997), para as tensões de 2, 4, 6, 10, 33, 100,500 e $1.500 \mathrm{kPa}$. As tensões de 2,4 e $6 \mathrm{kPa}$ foram obtidas utilizando-se a unidade de sucção (Grohmann, 1960), e, para obtenção das demais tensões, utilizou-se a câmara de Richards (Klute, 1986). Os valores de umidade gravimétrica e potencial matricial foram ajustados ao modelo proposto por van Genuchten (1980) para obtenção da curva de retenção:

$$
\theta=\theta_{r}+\frac{\theta s-\theta r}{\left[1+(\alpha \psi)^{n}\right]^{m}}
$$

em que $\theta_{r}\left(\mathrm{~cm}^{3} \mathrm{~cm}^{-3}\right)$ é o conteúdo de água residual; $\theta_{S}$ $\left(\mathrm{cm}^{3} \mathrm{~cm}^{-3}\right)$, o conteúdo de água de saturação; $\psi(\mathrm{cm})$, a tensão de água no solo; e $\alpha, m$ e $n$, parâmetros de ajuste do modelo.

As amostras previamente submetidas à tensão de $6 \mathrm{kPa}$ foram utilizadas na determinação da microporosidade (Grohmann, 1960). A porosidade total (PT) foi determinada por meio da umidade volumétrica do solo saturado, e a macroporosidade, calculada pela 
diferença entre a PT e a microporosidade. Foi calculada também a quantidade de água disponível (AD) $\left(\mathrm{m}^{3} \mathrm{~m}^{-3}\right)$, que foi obtida por diferença entre o teor de água do solo na capacidade de campo $\left(\theta_{c c}\right)$ e no ponto de murcha permanente $\left(\theta_{\mathrm{pmp}}\right)$, conforme a equação: $\left[\mathrm{AD}=\theta_{\mathrm{cc}}(6 \mathrm{kPa})-\theta_{\mathrm{pmp}}(1.500 \mathrm{kPa})\right]$ (Reichardt, 1988; Oliveira et al., 2003).

Em laboratório, o excedente das amostras indeformadas foi retirado de tal forma que o volume da amostra correspondesse ao volume do anel. Após o preparo das amostras indeformadas em laboratório, elas foram submetidas ao ensaio de compressão uniaxial (Bowles, 1986; Dias Junior, 1994), para obtenção das curvas de compressão do solo. As pressões aplicadas a cada amostra, utilizando um consolidômetro da marca Boart Longyear ${ }^{\circledR}$, obedeceram à seguinte ordem; 25, 50, 100, 200, 400, 800 e $1.600 \mathrm{kPa}$. Cada pressão foi aplicada até que 90 \% da deformação máxima fosse alcançada (Taylor, 1948), e somente então aplicava-se nova pressão. Após o término do ensaio, as amostras foram levadas à estufa a $105-110{ }^{\circ} \mathrm{C}$, até que se obtivesse massa constante, para determinação da umidade gravimétrica, conforme Embrapa (1997).

A partir das curvas de compressão foram obtidas as $\sigma_{\mathrm{p}}$, determinadas de acordo com Dias Junior \& Pierce (1995). Com a utilização do software Sigma Plot $10.0^{\circledR}$ (2002), foram obtidas as equações matemáticas que correspondessem aos modelos de capacidade de suporte de carga do solo, de acordo com o modelo $\sigma_{p}=10^{(a+b U)}$. As comparações das equações dos modelos de capacidade de suporte de carga entre os locais foram feitas adotando-se o procedimento descrito por Snedecor \& Cochran (1989).

A partir das amostras deformadas foram obtidos os teores de matéria orgânica (Raij \& Quaggio, 1983) e densidade de partículas (Blake \& Hartge, 1986). Os limites de liquidez (LL) e de plasticidade (LP) foram determinados conforme Sowers (1965), enquanto o limite de contração (LC) foi determinado segundo Bowles (1986).

Os valores referentes a porosidade total, macro e microporos e densidade do solo foram submetidos à análise exploratória, para obtenção da distribuição de freqüência e normalidade dos dados pelo teste $\mathrm{W}$ (Shapiro-Wilk, 1965). Nas situações em que ocorreu normalidade dos dados, procedeu-se à análise de variância e à comparação das médias, utilizando o teste de Scott-knott a $5 \%$, pelo programa estatístico Sisvar (Ferreira, 2000).

\section{RESULTADOS E DISCUSSÃO}

Os modelos de capacidade de suporte de carga $\left[\sigma_{\mathrm{p}}=10^{(\mathrm{a}+\mathrm{bU})}\right]$ para as diferentes posições de amostragem da lavoura cafeeira e o da mata nativa estão apresentados na figura 1. Os valores de "a" (intercepto da regressão linearizada) variaram de 2,65 a 2,68 , e os de "b" (coeficiente angular da regressão linearizada), de $-0,64$ a $-0,89$. Os coeficientes de determinação foram todos significativos a $1 \%$ pelo teste de t-Student e variaram de 0,78 a 0,84.

Para verificar as possíveis alterações estruturais do LVd causadas pelos diferentes usos e nas diferentes posições de amostragem na lavoura cafeeira, os modelos de capacidade de suporte de carga foram comparados (Quadro 1).

Observa-se que ambos os parâmetros dos modelos de CSC foram alterados (Quadro 1). Peng et al. (2004), estudando a relação entre pressão de preconsolidação e umidade gravimétrica, obtiveram um modelo exponencial semelhante ao utilizado neste estudo. Esses autores concluíram que o parâmetro "a" indica a resistência intrínseca do solo seco e o "b" descreve a influência de atributos do solo, como textura e matéria orgânica, na resistência deste. Entretanto, destaca-se

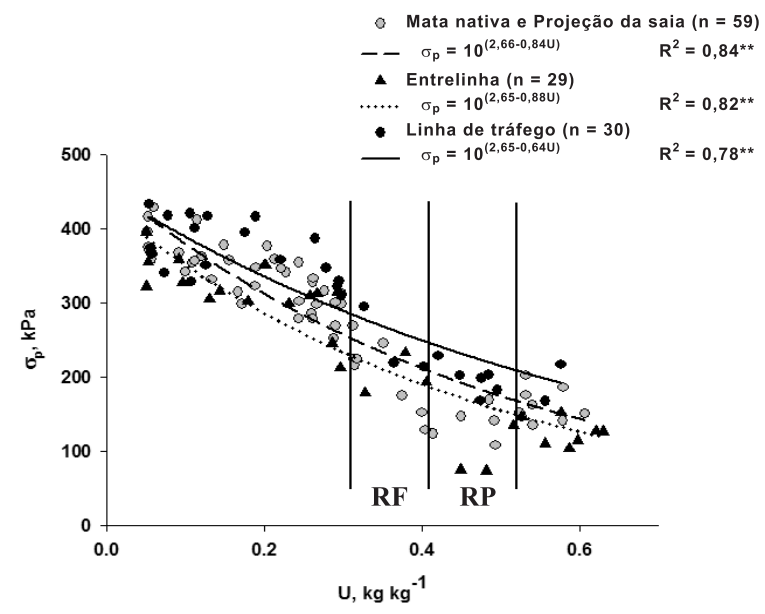

Figura 1. Modelos de capacidade de suporte de carga para mata nativa e projeção da saia, entrelinha e linha de tráfego. RF: região de friabilidade; RP: região de plasticidade.

Quadro 1. Teste de significância, descrito por Snedecor \& Cochran (1989), entre os modelos de capacidade de suporte de carga $\left[\sigma_{p}=10^{(a+b U)}\right]$ para o Latossolo Vermelho distrófico, para os diferentes locais na lavoura cafeeira e mata nativa

\begin{tabular}{lccc}
\hline & & \multicolumn{2}{c}{$\mathbf{F}$} \\
\cline { 3 - 4 } Localização & F & \multicolumn{2}{c}{ Coeficiente angular, b } \\
& & ns & a \\
\hline PS x MN & H & $*$ & ns \\
PS e MN x LT & H & ns & $*$ \\
PS e MN x EL & H & $*$ & $* *$ \\
LT x EL & H & & \\
\hline
\end{tabular}

PS: projeção da saia, MN: mata nativa; LT: linha de tráfego; EL: centro da entrelinha. ** $\mathrm{e}^{*}$ : significativo a 1 e $5 \%$, respectivamente; H: homogêneo; ns: não-significativo. 
que os autores utilizaram amostras deformadas e as submeteram ao ensaio de compressão uniaxial. No presente estudo, os parâmetros dos modelos de CSC foram independentes da textura e do teor de matéria orgânica, alterando-se de acordo com o aumento na história de tensão, aumentando os valores de "a" e reduzindo os de "b".

Os modelos de CSC do LVd, na projeção da saia do cafeeiro, não diferiram quanto à CSC em relação ao solo sob mata nativa, indicando uma homogeneidade na CSC da estrutura (Quadro 1). Devido a isso, uma nova equação foi ajustada, considerando-se todos os valores de pressão de preconsolidação e umidade, para a MN e PS, obtendo-se um único modelo de CSC (Figura 1).

A projeção da saia, entrelinha e linha de tráfego apresentaram comportamentos distintos com relação à capacidade de suporte de carga, devido aos manejos diferenciados aplicados em cada local. Nesse caso, entre os fatores que influenciam a capacidade de suporte de carga de um solo, o manejo (história de tensão) destaca-se, justificando esse comportamento. A EL apresentou menor capacidade de suporte de carga em razão do processo de subsolagem realizado 14 meses antes da data de amostragem (janeiro de 2006), apagando toda a história de tensão existente nesse local da lavoura cafeeira. A LT possui uma história de tensão induzida pelo tráfego desde a implantação da lavoura, apresentando maior capacidade de suporte de carga. APS mostrou comportamento intermediário quanto à CSC, refletindo a condição inicial de implantação da lavoura cafeeira, visto que sua CSC não diferiu da CSC do solo sob a MN (Quadro 1 e Figura 1).

Apesar de Rena \& Guimarães (2000) relatarem que solos com grande resistência mecânica podem ser benéficos à trafegabilidade de máquinas para o manejo da lavoura cafeeira, eles podem alterar severamente o crescimento do sistema radicular e a absorção de água e nutrientes pelas raízes. Isso não foi verificado na PS, onde se encontra a maior parte das raízes absorventes, pois o solo sob a posição PS mostrou o mesmo comportamento da MN quanto à capacidade de suporte de carga, revelando boas condições estruturais para crescimento radicular.
A região de friabilidade ( $\mathrm{RF})$ é considerada a faixa de umidade adequada para o preparo do solo (Hillel, 1982). As faixas de pressão que podem ser aplicadas aos solos na zona de friabilidade, com umidade na faixa de 0,31 a $0,41 \mathrm{~kg} \mathrm{~kg}^{-1}$, variaram de 207 a $251 \mathrm{kPa}$ para MN e PS, de 194 a $238 \mathrm{kPa}$ para EL e de 244 a $283 \mathrm{kPa}$ para LT (Figura 1). Desse modo, pressões aplicadas ao solo maiores do que esses limites poderão provocar compactação adicional mesmo na faixa de friabilidade, pelo fato de a capacidade de suporte de carga do solo ser excedida (Kondo \& Dias Junior, 1999).

Apesar de o solo na EL apresentar distribuição de poros e densidade do solo semelhantes às do solo na condição de MN (Quadro 2), esta apresenta maior CSC do que o solo na EL, devido à mobilização do solo pela subsolagem realizada na EL.

Os teores de matéria orgânica não diferiram estatisticamente entre as posições de amostragem avaliadas (Quadro 2). Assim, não é possível afirmar que o teor de matéria orgânica foi o fator que proporcionou a maior capacidade de suporte de carga do solo da MN, quando comparado com a EL. De acordo com Lebert \& Horn (1991), a pressão de preconsolidação do solo depende não somente de atributos como densidade e umidade do solo, mas também de fatores relacionados com sua estrutura, como a sua agregação. Silva \& Cabeda (2006) verificaram correlação positiva entre resistência dos agregados à ruptura e pressão de preconsolidação, comprovando sua influência na capacidade de suporte de carga do solo.

Na LT e PS, observa-se redução significativa da PT e da macroporosidade e aumento da microporosidade em comparação com a EL e MN (Quadro 2), corroborando os resultados obtidos por Lipiec \& Hatano (2003) e Yavuzcan et al. (2004). A redução dos macroporos deveu-se ao colapso da estrutura do solo, reduzindo o volume dos poros de maior tamanho devido à carga excessiva aplicada ao solo pelas operações mecanizadas, principalmente na LT. Desse modo, a ocorrência do processo de compactação pode causar deformação dos agregados do solo, reduzindo os poros de maior tamanho. A

Quadro 2. Propriedades físico-hídricas do Latossolo Vermelho distrófico típico, utilizando média de seis repetições

\begin{tabular}{|c|c|c|c|c|c|c|c|}
\hline Local & $\mathbf{P T}$ & Ma & Mi & $\mathrm{AD}$ & $\mathrm{Ma} / \mathrm{Mi}$ & Ds & MO \\
\hline & \multicolumn{4}{|c|}{$\mathrm{m}^{3} \mathrm{~m}^{-3}$} & & $\mathrm{Mg} \mathrm{m}^{-3}$ & $\mathrm{~g} \mathrm{~kg}^{-1}$ \\
\hline EL & $0,66 \mathrm{a}$ & $0,30 \mathrm{a}$ & $0,36 \mathrm{~b}$ & 0,11 a & $0,82 \mathrm{a}$ & $0,87 \mathrm{~b}$ & $35 \mathrm{a}$ \\
\hline PS & $0,63 \mathrm{~b}$ & $0,24 \mathrm{~b}$ & $0,39 \mathrm{a}$ & $0,11 \mathrm{a}$ & $0,62 \mathrm{~b}$ & $0,97 \mathrm{a}$ & $33 \mathrm{a}$ \\
\hline $\mathrm{LT}$ & $0,61 \mathrm{~b}$ & $0,21 \mathrm{~b}$ & $0,40 \mathrm{a}$ & $0,10 \mathrm{~b}$ & $0,54 \mathrm{~b}$ & $1,01 \mathrm{a}$ & $33 \mathrm{a}$ \\
\hline $\mathrm{MN}$ & $0,65 \mathrm{a}$ & $0,29 \mathrm{a}$ & $0,36 \mathrm{~b}$ & $0,10 \mathrm{~b}$ & $0,81 \mathrm{a}$ & $0,90 \mathrm{~b}$ & $36 \mathrm{a}$ \\
\hline
\end{tabular}

EL: entrelinha; PS: projeção da saia; LT: linha de tráfego; MN: mata nativa; PT: porosidade total; Ma: macroporosidade; Mi: microporosidade; AD: água disponível; Ds: densidade do solo; MO: matéria orgânica. Médias seguidas da mesma letra em cada coluna não diferem entre si pelo teste de Scott-Knott a $5 \%$. 
quebra dos agregados produz fragmentos que preenchem os macroporos, criando um espaço adicional de microporos (Starsev \& McNabb, 2001). De acordo com Kiehl (1979), um solo em condições ideais deve apresentar um terço da porosidade total formada por macroporos e os dois terços restantes por microporos, estabelecendo uma relação macroporos/ microporos igual a 0,5 , valor próximo ao encontrado nos locais mais compactados da lavoura (LT e PS). A maioria das plantas cresce satisfatoriamente seu sistema radicular quando a percentagem de macroporos está acima de $0,10 \mathrm{~m}^{3} \mathrm{~m}^{-3}$ (Kiehl, 1979; Gupta \& Allmaras, 1987), condição verificada no solo em todos os locais estudados (Quadro 2).

A distribuição de poros da LT e PS não foi diferente estatisticamente (Quadro 2), sugerindo um mesmo comportamento da curva de retenção de água nesses dois locais (Figura 2).

As posições LT e PS, em comparação com a MN (Quadro 2), tiveram redução na macroporosidade e aumento na microporosidade, que é a porosidade de retenção de água, concordando com Oliveira et al. (2004). Essa redução na PT condiciona maiores valores de densidade do solo observados nesses locais da lavoura cafeeira. Nota-se, portanto, que LT e PS apresentaram valores de macroporosidade e relação macro/microporosidade próximos da faixa considerada ideal por Kiehl (1979). De acordo com Resende et al. (2007), para Latossolos mais intemperizados, com baixa capacidade de armazenamento de água para as plantas, a compactação do solo poderia ser benéfica em termos de retenção de água, dentro de certos limites, pela transformação de parte dos macroporos em microporos.

Verifica-se que as curvas de retenção de água no solo foram influenciadas pelo processo de compactação (Figura 2), corroborando os resultados obtidos por Dias Junior \& Estanislau (1999). As curvas de retenção de água para o solo sob a LT e PS, nas tensões superiores a $6 \mathrm{kPa}$, foram deslocadas para a direita em relação a obtida para a $\mathrm{MN}$, proporcionando acréscimo da retenção de água nos maiores potenciais. Desse modo, observam-se maiores valores de umidade residual nesses locais, que é a umidade obtida quando se aplicam ao solo valores de suç̧ão de $1.500 \mathrm{kPa}$.

Esse fato é explicado pela redução do potencial de superfície causado pela compressão do solo, aumentando os pontos de contato entre partículas e, conseqüentemente, a adsorção da água (Assouline et al., 1997). Os locais onde há menor espaço poroso apresentaram menor conteúdo de água volumétrico em baixas tensões e maior conteúdo de água em tensões mais altas, o que pode ser observado na curva de retenção de água (Figura 2). Isso se deve ao maior volume de microporos em relação aos locais não alterados pelo processo de compactação do solo (Quadro 2).

Apesar dos valores semelhantes de água disponível $(\mathrm{AD})$, houve diferença estatística entre os locais

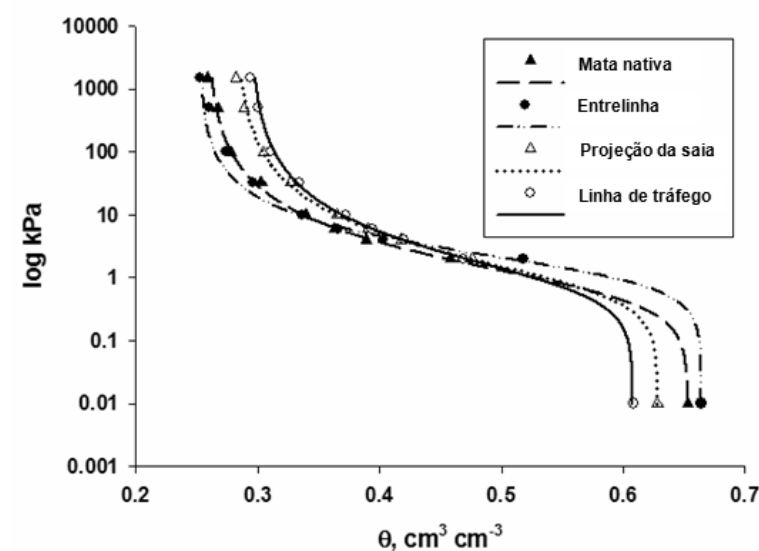

Figura 2. Curvas de retenção de água para um Latossolo Vermelho distrófico sob mata nativa e diferentes posições de amostragem na lavoura cafeeira.

avaliados, com EL e PS apresentando valores maiores em relação a LT e MN. Esse fato é importante, pois é na PS que se concentra a maioria das raízes absorventes, o que possibilita o maior aproveitamento de água pelos cafeeiros em períodos de maior déficit hídrico.

\section{CONCLUSÕES}

1. A capacidade de suporte de carga do solo decresce na seguinte ordem: $\mathrm{LT}>\mathrm{MN}=\mathrm{PS}>\mathrm{EL}$. A posição EL foi mais suscetível à compactação devido a uma subsolagem realizada meses antes da amostragem, reduzindo a resistência mecânica do solo.

2. AMN e a PS apresentaram a mesma capacidade de suporte de carga, indicando a manutenção de uma estrutura favorável para o crescimento do sistema radicular do cafeeiro.

3. A água disponível foi maior para as posições EL e PS e menor para a LT e MN; o solo sob a LT mantém maior microporosidade em relação à EL desestruturada pela subsolagem.

\section{LITERATURA CITADA}

ARAUJO-JUNIOR, C.F.; DIAS JUNIOR, M.S.; GUIMARÃES, P.T.G. \& PIRES, B.S. Resistência à compactação de um Latossolo cultivado com cafeeiro, sob diferentes sistemas de manejo de plantas invasoras. R. Bras. Ci. Solo, 32:23$32,2008$.

ASSOULINE, S.; TAVARES FILHO, J. \& TESSIER, D. Effect of compaction on soil physical and hydraulic properties: Experimental results and modeling. Soil Sci. Soc. Am. J., 61:390-398, 1997. 
BLAKE, G.F. \& HARTGE, K.H. Particle density. In: KLUTE, A., ed. Methods of soil analysis. Physical and mineralogical methods. 2.ed. Madison, American Society Agronomy, 1986. Part 1. p.377-382. (Agronomy Monograph, 9)

BOWLES, J.E. Engineering properties of soils and their measurements. 3.ed. New York, McGraw-Hill, 1986. 218 p.

CARVALHO FILHO, A.; DA SILVA, R.P. \& FERNANDES, A.L.T. Compactação do solo em cafeicultura irrigada. Uberaba, Universidade de Uberaba, 2004. 44p. (Boletim Técnico, 3)

DAY, P.R. Particle fractionation and particle-size analysis. In: BLACK, C.A., ed. Methods of soil analysis. Physical and mineralogical methods. 2.ed. Madison, American Society Agronomy, 1965. Part I. p.545-567. (Agronomy Monograph, 9)

DEXTER, A.R. Soil physical quality. Part I. Theory, effects of soil texture, density, and organic matter, and effects on root growth. Geoderma, 120:201-214, 2004.

DIAS JUNIOR, M.S. Compactação do solo. In: NOVAIS, R.F.; ALVAREZ V., V.H. \& SCHAEFER, C.E., eds. Tópicos em ciência do solo. Viçosa, MG, Sociedade Brasileira de Ciência do Solo, 2000. v.1. p.55-94.

DIAS JUNIOR, M.S. Compression of three soils under longterm tillage and wheel traffic. East Lansing, Michigan State University, 1994. 114p. (Tese de Doutorado)

DIAS JUNIOR, M.S. \& ESTANISLAU, W.T. Grau de compactação e retenção de água de Latossolos submetidos a diferentes sistemas de manejo. R. Bras. Ci. Solo, 23:45$51,1999$.

DIAS JUNIOR, M.S. \& PIERCE, F.J. O processo de compactação do solo e sua modelagem. R. Bras. Ci. Solo, 20:175-182, 1996.

DIAS JUNIOR, M.S. \& PIERCE, F.J. A simple procedure for estimating preconsolidation pressure from soil compression curves. Soil Technol., 8:139-151, 1995.

EMPRESA BRASILEIRA DE PESQUISA AGROPECUÁRIA . EMBRAPA. Centro Nacional de Pesquisas de Solos. Manual de métodos de análise de solo. Rio de Janeiro, 1997. $212 \mathrm{p}$

EMPRESA BRASILEIRA DE PESQUISA AGROPECUÁRIA EMBRAPA. Centro Nacional de Pesquisas de Solos. Sistema brasileiro de classificação de solos. 2.ed. Rio de Janeiro, Embrapa Solos, 2006. 306p.

FERREIRA, D.F. Análises estatísticas por meio do SISVAR para Windows 4. 0. In: REUNIÃO ANUAL DA REGIÃO BRASILEIRA DA SOCIEDADE INTERNACIONAL DE BIOMETRIA, 45., 2000, São Carlos. Anais. São Carlos, Universidade Federal de São Carlos, 2000. p.255-258.

FLOWERS, M. \& LAL. R. Axle load and tillage effect on soil physical properties and soybean grain yield on a Mollic Ochraqualf in Northwest Ohio. Soil Till. Res., 48:21-35, 1998.

GROHMANN, F. Distribuição e tamanho de poros em três tipos de solos do Estado de São Paulo. Bragantia, 19:319. $328,1960$.
GUPTA, S.C. \& ALLMARAS, R.R. Models to assess the susceptibility of soils to excessive compaction. Adv. Soil Sci., 6:5-10, 1987.

HILLEL, D. Introdution to soil physics. San Diego, Academic, 1982. 365p.

HILLEL, D. Solo e água: Fenômenos e princípios físicos. Traduzido pelo convênio UFRGS-USAID/ Wisconsin. Porto Alegre, Universidade Federal do Rio Grande do Sul, 1970. 231p.

KIEHL, E.L. Manual de edafologia. São Paulo, Ceres, 1979. $262 \mathrm{p}$.

KLUTE, A. Laboratory measurement of hydraulic conductivity of saturated soils. Madison, American Society of Agronomy, 1986. p.253-261 (Monograph, 9)

KONDO, M.K. \& DIAS JUNIOR, M.S. Compressibilidade de três Latossolos em função da umidade e uso. R. Bras. Ci. Solo, 23:211-218, 1999.

LEBERT, M. \& HORN, R. A method to predict the mechanical strength of agricultural soils. Soil Till. Res., 19:275-286, 1991.

LIPIEC, J. \& HATANO, R. Quantification of compaction effects on soil physical properties and crop growth. Geoderma, 116:107-136, 2003.

OLIVEIRA, G.C.; DIAS JUNIOR, M.S.; CURI, N. \& RESCK, D.V.S. Caracterização química e físico-hídrica de um Latossolo Vermelho após vinte anos de manejo e cultivo do solo. R. Bras. Ci. Solo, 28:327-336, 2004.

OLIVEIRA, G.C.; DIAS JUNIOR, M.S.; RESCK, D.V.S. \& CURI, N. Alterações estruturais e comportamento compressivo de um Latossolo Vermelho distrófico argiloso sob diferentes sistemas de uso e manejo. Pesq. Agropec. Bras., 38:291-299, 2003.

PENG, S.H.; HORN, R.; ZHANG, B. \& ZAHO, Q.G. Mechanisms of soil vulnerability to compaction of homogenized and recompacted Ultisols. Soil Till. Res., $76: 125-137,2004$

PIRES, L.F.; CÁSSARO, F.A.M.; REICHARDT, K. \& BACCHI, O.O.S. Soil porous system changes quantified by analysing soil water retention curve modifications. Soil Till. Res., 100:72-77, 2008.

RAIJ, B.van \& QUAGGIO, J.A. Métodos de análises de solo para fins de fertilidade. Campinas, Instituto Agronômico de Campinas, 1983. 31p. (Boletim Técnico, 81)

REICHARDT, K. Capacidade de campo. R. Bras. Ci. Solo, 12:211-216, 1988

RENA, A.B. \& GUIMARÃES, P.T.G. Sistema radicular do cafeeiro: Estrutura, distribuição, atividades e fatores que o influenciam. Belo Horizonte, Epamig, 2000. 80p. (Epamig. Série Documentos, 37)

RESENDE, M.; CURI, N.; REZENDE, S.B. \& CORREAA, G.F. Pedologia: Base para distinção de ambientes. 5.ed. Lavras, Universidade Federal de Lavras, 2007. 322p. 
SÁ, M.A.; LIMA, J.M. \& LAGE, G. Procedimento padrão para medida da potência liberada pelo aparelho ultra-som. Ci. Agrotec., 24:300-306, 2000 .

SHAPIRO, S.S. \& WILK, M.B. An analysis of variance test for normality: Complete samples. Biometrika, 52:591-611, 1965.

SIGMA PLOT. Scientific Graphing Software. Versão 8.0. Software. San Rafael, Jandel Corporation, 2002.

SILVA, A.J. \& CABEDA, M.S.V. Compactação e compressibilidade do solo sob sistemas de manejo e níveis de umidade. R. Bras. Ci. Solo, 30:921-930, 2006.

SILVA, A.R.; DIAS JUNIOR, M.S.; GUIMARÃES, P.T.G. \& ARAUJO JUNIOR, C.F. Modelagem da capacidade de suporte de carga e quantificação dos efeitos das operações mecanizadas em um Latossolo Amarelo cultivado com cafeeiros. R. Bras. Ci. Solo, 30:207-216, 2006.

SILVA, E.M. \& MALVINO, S.S.A.B. Análise climática do município de Patrocínio (MG). Caminhos da geografia, 10:93-108, 2005. Disponível em: <www. ig. ugu. br/revista/ caminhos. html>.

SNEDECOR, G.W. \& COCHARAN, W.G. Statistical methods. 8.ed. Ames, Iowa State University, 1989. 503p.
SOWERS, G.F. Consistency. In: BLACK, C.A., ed. Methods of soil analisys: Physical and mineralogical properties including statistics of measurement and sampling. Madison, American Society of Agronomy, 1965. pt. 1. p.391-399.

STARTSEV, A.D. \& McNABB, D.H. Skidder traffic effects on water retention, pore-size distribution, and van Genuchten Parameters of Boreal forest soils. Soil Sci. Soc. Am. J., 65:224-231, 2001.

TAYLOR, D.W. Fundamentals of soil mechanics. New York, J. Wiley, 1948. 700p.

TIMM, L.C.; PIRES, L.F.; ROVERATTI, R.; ARTHUR, R.C.J.; REICHARDT, K.; OLIVEIRA, J. C.M. \& BACCHI, O.O.S. Field spatial and temporal patterns of soil water content and bulk density changes. Sci. Agric., 63:55-64, 2006.

TIMM, L.C.; PIRES, L.F.; REICHARDT, K.; ROVERATTI, R.; OLIVEIRA, J.C.M. \& BACCHI, O. O.S. Soil bulk density evaluation by conventional and nuclear methods. Aust. J. Soil Res., 43:97-103, 2005.

van GENUCHTEN, M.T. A closed-form equation for predicting the hydraulic conductivity of unsaturated soils. Soil Sci. Soc. Am. J., 44:892-898, 1980.

YAVUZCAN, H.G.; MATTHIES, D. \& AUERNHAMMER, H. Vulnerability of Bavarian silty loam soil to compaction under heavy wheel traffic: Impacts of tillage method and soil water content. Soil Till. Res., 84:200-215, 2005. 